\title{
émulations
}

Lilian Mathieu

2018

\section{La traite des êtres humains, de la légende urbaine à la politique publique}

\begin{abstract}
Avertissement
Le contenu de ce site relève de la législation belge sur la propriété intellectuelle et est la propriété exclusive de l'éditeur.

Les œuvres figurant sur ce site peuvent être consultées et reproduites sur un support papier ou numérique sous réserve qu'elles soient strictement réservées à un usage soit personnel, soit scientifique ou pédagogique excluant toute exploitation commerciale. La reproduction devra obligatoirement mentionner l'éditeur, le nom de la revue, l'auteur et la référence du document.

Toute autre reproduction est interdite sauf accord préalable de l'éditeur, en dehors des cas prévus par la législation en vigueur en Belgique.
\end{abstract}

\section{Référence électronique}

Citation : Lilian Mathieu, «La traite des êtres humains, de la légende urbaine à la politique publique », Émulations, en ligne. Mise en ligne le 7 mai 2018. URL : http://www.revue-emulations.net/enligne/Mathieu-traite-des-etres-humains

Éditeur : Émulations - Revue de sciences sociales http://www.revue-emulations.net 


\section{La traite des êtres humains, de la légende urbaine à la politique publique}

Lilian Mathieu

CNRS, Centre Max Weber-École normale supérieure de Lyon

\section{Résumé/Abstract}

[Fr] La traite des blanches (requalifiée aujourd'hui en traite des êtres humains) constitue le motif de l'une des légendes urbaines les plus largement diffusées et les mieux étudiées, mettant en scène une jeune fille droguée puis enlevée pour être contrainte à la prostitution dans un pays étranger. Mais la traite est aussi, et de longue date, l'objet de politiques publiques spécifiques, légitimées par des conventions internationales et mises en œuvre par la police et la justice. Cette coexistence d'une légende urbaine et d'une politique publique constitue une énigme, que l'article tente de résoudre : comment imaginer que des moyens considérables soient déployés contre des faits à la réalité aussi incertaine ? Une analyse historique et sociologique révèle que croyances populaires et discours institutionnels se sont mutuellement consolidés, chacun apportant sa contribution spécifique à la plausibilité du motif de la traite.

Mots clés : traite des êtres humains, légende urbaine, politique publique, prostitution, abolitionnisme.

[En] The White slave trade (nowadays known as human trafficking) is the motif of one of the most well-known and well-studied urban legends: a young girl is drugged before being taken away and forced into prostitution in a foreign country. But trafficking of human beings is also, and has been for a long time, the object of specific public policies that have been legitimated by international conventions and enforced by both police and justice. The coexistence of an urban legend and of a public policy stands as an enigma that this article intends to solve: how can one imagine that important means are mobilised against so dubious facts? A historical and sociological analysis reveals that popular beliefs and institutional stances have mutually consolidated, each one giving weight to the plausibility of the motif of the trade.

Keywords: trafficking of human beings, urban legend, public policy, prostitution, abolitionism. 


\section{Introduction}

La traite des blanches (requalifiée aujourd'hui en traite des êtres humains) constitue le motif de l'une des légendes urbaines les plus largement diffusées et les mieux étudiées. Une de ses déclinaisons classiques met en scène une jeune fille qui, essayant des vêtements dans une cabine d'essayage, est droguée afin d'être ensuite livrée à un réseau de trafiquants qui la destine à la prostitution dans un pays étranger. Clé de son «succès », la structure fantasmatique du récit est fortement genrée et érotisée : un personnage féminin posé comme vulnérable (par sa jeunesse mais aussi son isolement, sa nudité et l'emprise d'un stupéfiant) devient la proie de figures malveillantes, implicitement posées comme masculines, qui la destinent à des pratiques sexuelles non consenties (et dont la nudité dans la cabine constitue une anticipation).

Mais la traite des êtres humains n'est pas qu'une légende urbaine récurrente dans les pays occidentaux. Elle est aussi, et de longue date, l'objet de politiques publiques spécifiques. Pour s'en tenir à ce seul pays, la France a ratifié en 1960 la Convention de l'ONU pour la répression de la traite des êtres humains et de l'exploitation de la prostitution d'autrui, a accueilli en 2003 dans son code pénal le nouveau délit de traite des êtres humains puni de sept ans de prison et $150000 €$ d'amende et a institué en 2013 une Mission interministérielle pour la protection des femmes victimes de violences et la lutte contre la traite des êtres humains (MIPROF). Des chiffres circulent, produits par les experts d'ONG ou d'institutions internationales, qui mesurent tant l'ampleur du phénomène que l'importance des bénéfices qu'il rapporte à ses organisateurs criminels (Mathieu, 2014 ; Chaumont, 2012).

La coexistence d'une légende urbaine et d'une politique publique constitue une énigme: comment imaginer que des moyens considérables soient déployés contre des faits à la réalité incertaine ? Jean-Michel Chaumont (2009) s'y est confronté en détaillant comment un groupe d'experts de la Société des nations (SDN) avait construit une traite fictionnelle à l'aune de leurs intérêts institutionnels et de leurs certitudes moralistes, alors même qu'ils disposaient d'informations plus concrètes et nuancées sur les logiques des migrations prostitutionnelles ${ }^{1}$. Ce qu'il désigne comme le «mythe », terme également utilisé par Corbin (1982 : 407), de la traite des blanches présenterait cette particularité, en regard de récits légendaires ancrés dans les milieux populaires, d'avoir été fabriqué au sein des couches privilégiées et diffusé de haut en bas, en abusant d'une autorité tout à la fois morale, intellectuelle et politique.

Résoudre l'énigme en renvoyant la traite du côté du mythe peut cependant laisser insatisfait car, le situant dans l'alternative du vrai et du faux, elle manque cet aspect essentiel qu'est la plausibilité du motif de la traite, gage de son efficacité sociale et politique. Le présent article ${ }^{2}$ entend soumettre l'unilatéralisme de la thèse de Chaumont à un examen critique : il est probable, d'une part, que le motif fictionnel diffusé par les experts de la SDN n'a pas été élaboré à partir de rien et, d'autre part, qu'il n'aurait pas rencontré un tel succès s'il n'avait pas conforté un fonds de croyances sur les risques qui pèsent sur la vertu des jeunes filles imprudentes. De fait, plutôt que s'opposer, la légende urbaine et la politique publique semblent s'être mutuellement consolidées en se confirmant et se renforçant l'une l'autre.

\section{Traite d'en haut, traite d'en bas ?}

Appréhendé dans sa dimension légendaire, le motif de la traite apparait comme une transposition moderne du conte du petit chaperon rouge (Doezema, 2010) : une héroïne

\footnotetext{
${ }^{1}$ Cette dimension est également au cœur du travail d'Alain Corbin (1982) qui atteste la réalité des circulations de prostituées entre les bordels de divers pays mais qui, constatant plutôt leur engagement délibéré dans ces migrations, réfute que celles-ci aient été nécessairement contraintes ou dupées par de vils proxénètes.

${ }^{2}$ Cet article s'appuie ici sur un travail bibliographique et une investigation archivistique menés dans le cadre d'une recherche sociologique consacrée au mouvement abolitionniste français (Mathieu, 2014).
} 
cumulant les traits de fragilité (féminité, jeunesse, naïveté, éventuellement beauté) entreprend un déplacement hors de son foyer qui, en l'isolant socialement (dans la forêt ou la cabine d'essayage), l'expose à un péril incarné par un personnage masculin (le loup, le proxénète) doté de pouvoirs supérieurs et désireux d'attenter à son intégrité physique (la manger ou l'asservir sexuellement).

Gary Fine (1992) a relevé que l'impersonnalité de la vie urbaine moderne occidentale offrait un terrain favorable à une transposition de ce motif à ces lieux anonymes que sont les centres commerciaux $^{3}$. Le phénomène est probablement aussi ancien que l'urbanisation, comme le suggère la rumeur, surgie à Paris au milieu du XVIII ${ }^{\mathrm{e}}$ siècle, selon laquelle les forces de l'ordre enlèveraient des enfants à leurs parents pour les livrer à des aristocrates afin que ceux-ci tentent de se guérir d'horribles maladies en se baignant dans leur sang (Farge, Revel, 1988). On reconnaît ici la vulnérabilité (les victimes sont définies par leur jeune âge) renforcée par l'isolement (les enfants sont enlevés alors qu'ils échappent à la surveillance de leurs parents) qui rend possible un asservissement à des individus aussi puissants (ce sont des nobles) que répugnants (leurs maladies témoignent d'une dégénérescence à la fois physique et morale) et l'atteinte corporelle atroce (la mort par saignée).

Le schéma d'analyse descendant de Chaumont ne s'applique guère ici. Le récit repose sur une structure hiérarchique qui oppose des dominants dégénérés et malfaisants à des dominés vulnérables. Il est en outre difficile de penser que sa diffusion ait pu servir les dominants puisque la rumeur déboucha sur une révolte au cours de laquelle un officier fut lynché. Le même ancrage au bas de l'échelle sociale est repérable dans ce qui constitue l'expression la plus classique - puisqu'elle fut l'objet d'une étude sociologique « à chaud » - du motif de la traite, à savoir la rumeur qui, au printemps 1969, incrimina les cabines d'essayage de magasins de vêtements orléanais tenus par des juifs. L'équipe réunie par Edgar Morin juste après l'acmé de la rumeur parvint à en reconstituer les étapes, circuits de diffusion et principaux ressorts fantasmatiques ${ }^{4}$. Elle identifia surtout le principal « milieu conducteur » de la panique dans une jeunesse à la fois féminine - que les résonnances aussi menaçantes qu'érotiques du motif ne pouvait laisser insensible - et dépourvue des instruments cognitifs de résistance aux rumeurs fantaisistes - ce que Morin qualifie de «zone de sous-culture politique » consommant « intensivement la culture de masse » (Morin, $1969: 81^{5}$ ).

Les médias de masse offrent sans doute une des clés permettant de surmonter l'opposition entre légendes distillées respectivement d'en haut (Chaumont) ou d'en bas (Morin) de l'échelle sociale. L'étude que livre Judith Walkowitz (1992) de ce qui fut la première cristallisation systématique du schème de la traite des blanches ${ }^{6}$ - à savoir la série d'articles sensationnalistes que publia William T. Stead en 1885 dans la Pall Mall Gazette - est à ce titre particulièrement éclairante. Le texte de Stead se réfère explicitement à un fonds mythologique - la légende antique du Minotaure - pour livrer le récit de l'enquête dans les bas-fonds de Londres qui lui aurait permis de découvrir qu' " au milieu de nous fonctionne un système dont le viol de vierges constitue un des incidents ordinaires ; [...] les arrangements pour procurer, certifier, violer,

\footnotetext{
${ }^{3}$ La version qu'il évoque comporte quelques traits distinctifs : ce sont les toilettes d'un centre commercial et non une cabine d'essayage qui isolent la victime de ses proches et elle est enlevée par deux femmes dont l'une prétend être sa mère ; mais elle est effectivement droguée et destinée à la traite des blanches.

4 À ceux déjà repérés (jeunesse des victimes féminines, isolement dans l'espace urbain, usage de drogues, prostitution comme destin, etc.) s'ajoute ici la figure du juif comme incarnation malfaisante, selon le schème antisémite classique articulant avidité et agissement occulte.

${ }^{5}$ Qu'en 2014 l'auteur de ces lignes ait reçu un écho de la rumeur d'Orléans auprès de lycéennes (qui souhaitaient l'interviewer pour un travail collectif sur le thème de la prostitution) d'une des institutions privées les plus huppées de Lyon inviterait à nuancer ou actualiser cette analyse.

${ }^{6}$ Elle fut précédée, quelques années auparavant, par l' «affaire des petites anglaises », un scandale né de la découverte que des jeunes anglaises, dont certaines mineures de moins de 21 ans, comptaient parmi les pensionnaires d'un bordel bruxellois «protégé » par la police belge (Chaumont, Machiels, 2009).
} 
entretenir et exploiter ces malheureuses victimes se font avec une simplicité et un succès incroyables » (Stead, $1885: 21$ ). Walkowitz souligne combien le texte de Stead parvient à combiner deux registres antithétiques, celui du récit pornographique et celui, très en vogue au sein du lectorat féminin du XIX ${ }^{\mathrm{e}}$ siècle, du mélodrame, en insistant sur des thèmes tels que « la poursuite masculine d'un objet de désir féminin qui transgresse les barrières de classe, l'association entre sexe et violence, et la représentation d'une sexualité masculine agressive s'imposant à une féminité passive et asexuée » (Walkowitz, 1992: 97). Il se singularise également en ce qu'il ne met pas seulement en cause les riches aristocrates prêts à acheter des jeunes filles pour les soumettre à leur débauche mais aussi les mères indignes du prolétariat prêtes à vendre leurs enfants.

Les ressorts narratifs mobilisés par Stead furent indéniablement efficaces. Véritable panique morale ${ }^{7}$, l'émoi public suscité par ses révélations impulsa une mobilisation de masse relayée par des entrepreneurs de morale puritains et déboucha sur une réforme législative élevant l'âge du consentement sexuel des filles de 13 à 16 ans, accordant des pouvoirs étendus à la police pour contrôler les bordels et réprimer la prostitution de rue, et instaurant une pénalisation aggravée des écrits obscènes comme de l'homosexualité. Le procès intenté contre Stead - qui, pour attester les faits qu'il dénonçait, avait lui-même entrepris d'acheter une fillette à sa mère - ne suffit pas à éteindre la conviction que les déplacements et les loisirs des jeunes filles des milieux populaires devaient être étroitement contrôlés pour mieux les protéger. Walkowitz souligne également combien le récit de Stead minore la dimension économique des faits qui l'indignent : plus que l'avidité de leurs mères ou la perversité des riches aristocrates, ce sont le chômage et la pauvreté endémiques qui amenaient tant de jeunes Anglaises à la prostitution. Cette fois, la thèse de Chaumont (2009) paraît validée : l'invocation d'enlèvements de jeunes filles à la réalité incertaine cautionne l'instauration de mesures attentatoires aux libertés individuelles, au service d'un conservatisme tant économique que moral. Mais il est à parier que l'écho des écrits de Stead tient en large partie à ce qu'ils sont venus conforter ou ratifier un fonds de croyances diffuses et brumeuses - on sait vaguement, car on l'a entendu dire de-ci delà, que des jeunes filles sont enlevées pour être livrées à des sévices sexuels, et une parole revêtue des apparats de l'autorité intellectuelle et morale en apporte confirmation.

\section{Mobilisations et institutionnalisations d'un motif légendaire}

Stead s'était trouvé une alliée en Josephine Butler, leader du mouvement pour l'abolition de la réglementation de la prostitution. La dénonciation de la traite des blanches a permis à l'abolitionnisme anglais de trouver un nouveau souffle au moment précis où, parvenu au succès avec l'abrogation du contrôle sanitaire obligatoire des prostituées, il risquait de se trouver sans cause à défendre (Mathieu, 2014 ${ }^{8}$ ). La Fédération abolitionniste internationale, fondée en 1875 et animée par Butler, se coalise avec divers apôtres de la décence publique pour mener campagne contre les déplacements internationaux de prostituées. C'est au travers du motif de la traite que ces migrations prostitutionnelles, favorisées tant par la pauvreté des jeunes femmes de milieu populaire que par les exigences de renouvellement du personnel des bordels, ont été publicisées par les abolitionnistes mais aussi appropriées par les autorités politiques de divers pays européens. S'est enclenché, dès le début du $\mathrm{XX}^{\mathrm{e}}$ siècle, un processus d'institutionnalisation de la traite des blanches, principalement sous la forme d'accords

\footnotetext{
${ }^{7}$ Charles Krinsky (2013) définit les paniques morales comme des épisodes durant lesquels un public ressent de la préoccupation, de l'anxiété, de la peur ou de la colère à l'égard de ce qu'il perçoit comme une menace pour l'ordre social.

${ }^{8}$ Le thème de la traite ne pouvait que rencontrer un écho au sein d'un mouvement directement issu de celui favorable à l'abolition de l'esclavage. La traite des blanches a de fait été construite par les abolitionnistes en homologie explicite avec la traite des Africains réduits en esclavage en Amérique.
} 
internationaux. Le premier instaure en 1901 un arrangement entre seize pays destiné à la lutte contre la «traite des filles mineures et des femmes majeures non consentantes » (par fraude, abus, abus d'autorité, violence, menace ou autre moyen de contrainte), principalement par un échange de renseignements entre pays, une surveillance des gares et des ports, des campagnes de mise en garde des jeunes filles ainsi qu'un rapatriement des victimes ${ }^{9}$.

D'autres textes suivront : en 1910, une convention internationale relative à la répression de la traite des blanches prévoit la pénalisation de l'embauche à des fins de prostitution d'une mineure de 20 ans, mais aussi d'une majeure (en cas de contrainte ou de ruse). En 1921 puis en 1933, c'est la Société des nations qui s'approprie la lutte contre la traite, en mettant en place une commission technique permanente dédiée, celle étudiée par Chaumont (2009), et en raffinant les moyens de lutter contre le fléau (la traite concerne progressivement les enfants sans distinction de sexe ainsi que les femmes adultes indépendamment de leur consentement, en prévoyant des mesures destinées à contrôler celles qui entreprennent de migrer seules). Après la Seconde Guerre mondiale, l'ONU ouvre à ratification la Convention pour la répression de la traite des êtres humains et de l'exploitation de la prostitution d'autrui, dont le préambule pose la traite comme indissociable de la prostitution. Dès 1958, deux ans avant de ratifier ce texte, l'État français se dote d'un instrument policier spécifiquement destiné à combattre la traite, l'Office central pour la répression de la traite des êtres humains (OCRTEH) qui, aujourd'hui encore, collecte toutes les données et mène des investigations de portée nationale et internationale sur les faits de traite.

Cette appropriation institutionnelle est soutenue par le mouvement abolitionniste français, pour qui la lutte contre la traite constitue une thématique centrale. Avant la Seconde Guerre mondiale, elle est associée à la dénonciation des bordels (accusés de recourir à la traite pour renouveler leurs effectifs) et reproduit la structure narrative centrée sur l'abus de la naïveté des futures victimes :

Les moyens par lesquels ces forbans parviennent à s'emparer de la proie sur laquelle ils ont jeté leur dévolu sont forts divers. Tantôt ce sera le racolage proprement dit, tantôt la promesse d'une situation illusoire, ou une promesse de fiançailles, parfois même le mariage effectivement célébré. Si la ruse ne réussit pas, ils ne craindront pas de recourir à la violence ou aux narcotiques pour avoir raison de la résistance de leurs victimes ${ }^{10}$.

Le motif ne disparaît pas après l'interdiction des maisons closes en 1946 et il est par exemple récurrent chez l'une des intellectuelles organiques du mouvement, Odette Philippon. Les écrits de cette auteure, qui se présente comme «enquêtrice sociale », regorgent d'histoires de jeunes filles naïves enlevées ou séduites puis vendues et forcées à la prostitution. Dans ce qui se veut un ouvrage à destination des jeunes (et spécialement des jeunes filles), Philippon met en garde devant tous les lieux périlleux pour une «jeunesse trop encline à l'indépendance » : les bals publics, les cinémas, les caves existentialistes, l'auto-stop et... les cabines d'essayage car «il se passerait derrière les rideaux [...] des obscénités » (Philippon, $1958: 30$ ).

Pourtant, la réalité du phénomène est toujours aussi difficile à saisir. Corbin fait remarquer que les outils juridiques mis en place au début du $\mathrm{XX}^{\mathrm{e}}$ siècle n'ont que rarement débouché sur des poursuites et encore moins sur des condamnations, ce qui selon lui «prouve l'importance des fantasmes en cette matière » (Corbin, 1982 : 434). L'étude des rapports annuels du chef de la section de police judiciaire du département du Rhône confirme que tel est toujours le cas à la fin des années 1940. Conformément à la définition très large et indécise de la traite posée par

\footnotetext{
${ }^{9}$ Le sujet fait dès 1903 l'objet d'une thèse de sciences politiques et économiques qui s'émeut de « l'embauchage de malheureuses et honnêtes filles, à qui des positions brillantes étaient promises à l'étranger et qui se trouvaient contraintes, arrivées à destination, de se livrer à la débauche »; l'auteur s'étonne plus loin de «l'excès de naïveté surprenant » des jeunes filles qui se laissent séduire par ces promesses (Appleton, 1903 : 10, 28).

${ }^{10}$ Les scandales de la prostitution réglementée, brochure de l'Union temporaire contre la prostitution réglementée et la traite des femmes, 1938, p. 13.
} 
une circulaire du ministère de l'Intérieur du 4 mai 1939 (« le terme "traite des femmes et des enfants" doit être considéré comme englobant [...] tous les aspects de la prostitution commercialisée »), les rapports recensent l'ensemble des délits associés à la prostitution (racolage, tenue de maison clandestine, proxénétisme, etc.) voire au-delà (détournements de mineur, attentats à la pudeur, actes impudiques sur mineur de même sexe, publications obscènes, etc.) mais ne signalent aucun fait de traite stricto sensu. Comme le rapporte Gwenaëlle Mainsant (2012: 105), le constat de l'absence d'enlèvement de jeunes filles destinées à la prostitution est confirmé au niveau national par un rapport de la brigade mondaine signalant en 1956 que les enquêtes pour traite «n'ont en général abouti qu'à établir, soit l'inexistence du "cas" signalé, soit une fugue volontaire n'ayant aucun rapport avec une affaire de proxénétisme ». La même année, pourtant, la députée du Mouvement républicain et populaire (MRP) Francine Lefebvre déclarait dans la presse abolitionniste que 100000 Françaises auraient été enlevées pour la traite des blanches ${ }^{11}$.

Le thème de la traite s'estompe du discours et des préoccupations du mouvement abolitionniste à compter des années 1960, notamment sous l'effet d'une relative disqualification, à laquelle le féminisme naissant a sans doute contribué, de la figure de la naïve jeune fille avilie par une sexualité masculine prédatrice ${ }^{12}$. Certes, la revue des Équipes d'action contre la traite des femmes et des enfants pouvait encore en 1979 mettre en garde «nos filles » contre l'auto-stop, les bals publics, les flirts avec des inconnus, les mariages hâtivement conclus ou les emplois de danseuse, strip-teaseuse ou modèle nu, posés en antichambres de la prostitution $^{13}$. Mais la même association, constatant la ringardise du motif de la traite, décidait au début des années 1990 de se rebaptiser «Équipes d'action contre le proxénétisme ».

\section{Renouveau et consécration}

Un tel choix a dû rétrospectivement paraître prématuré et malencontreux aux Équipes d'action puisqu'on assiste précisément à la fin des années 1990 à un renouveau spectaculaire du thème de la traite. Celui-ci est dû à l'action combinée de plusieurs facteurs. Le premier est une nouvelle actualité institutionnelle de la traite, consacrée par le protocole additionnel - dit « de Palerme » - à la Convention des Nations Unies contre la criminalité transnationale organisée, ouvert à signature en 2000, qui définit la traite des êtres humains comme :

Le recrutement, le transport, le transfert, l'hébergement ou l'accueil de personnes, par la menace de recours ou le recours à la force ou à d'autres formes de contrainte, par enlèvement, fraude, tromperie, abus d'autorité ou d'une situation de vulnérabilité, ou par l'offre ou l'acceptation de paiements ou d'avantages pour obtenir le consentement d'une personne ayant autorité sur une autre aux fins d'exploitation (dont l'exploitation de la prostitution d'autrui ou d'autres formes d'exploitation sexuelle ${ }^{14}$. L'adoption de ce texte témoigne certes d'une préoccupation de la communauté internationale devant le développement de la criminalité organisée mais également du nouvel enjeu de politique internationale que représente le contrôle des migrations.

La nouvelle articulation entre migration féminine et prostitution constitue précisément le deuxième élément favorable à la réémergence de la traite. Celle-ci se manifeste, à la même

\footnotetext{
${ }^{11}$ Moissons nouvelles, $\mathrm{n}^{\circ} 18,1956$ (il s'agit du journal de la principale association abolitionniste française, le Mouvement du Nid, lequel regorge de récits mélodramatiques de jeunes filles pauvres que leur crédulité conduit à la prostitution, souvent après avoir été séduites puis abandonnées à une maternité hors mariage).

12 Il n'est pas impossible que l'écho du livre de Morin (1969), posant explicitement le statut légendaire des enlèvements de jeunes filles contraintes à la prostitution, ait lui aussi contribué à disqualifier le motif de la traite. Comme on verra plus loin, son renouveau contemporain fait de la naïveté un trait propre aux jeunes femmes « étrangères ».

${ }^{13}$ Esclavage, $\mathrm{n}^{\circ} 25,1979$.

${ }^{14}$ Convention des Nations Unies contre la criminalité transnationale organisée et protocoles d'y rapportant, Office des Nations Univers contre la drogue et le crime, Vienne, 2004, p. 42-43. En ligne, consulté le 18 avril 2018. URL : http://www.unodc.org/documents/treaties/UNTOC/Publications/TOC\%20Convention/TOCebook-f.pdf.
} 
époque, dans la présence visible de jeunes femmes étrangères (dans un premier temps originaires d'Europe de l'Est puis d'Afrique sub-saharienne et dans une moindre mesure d'Asie) sur les trottoirs des grandes villes. La mobilisation du schème de la traite pour rendre compte de leur arrivée lui a fait subir un complet renversement : alors que celui-ci désignait autrefois le déplacement de jeunes Françaises vers l'étranger, ce sont désormais des étrangères qui sont amenées en France pour y être prostituées. La victime de la traite n'en reste pas moins définie par une vulnérabilité foncière, que signalent à la fois sa jeunesse et son altérité culturelle.

Les associations abolitionnistes vont s'emparer de cette nouvelle réalité prostitutionnelle pour s'engager dans une remobilisation d'autant plus vigoureuse qu'elle peut compter, à la fois, sur le soutien de fractions conséquentes des mouvements féministe et altermondialiste, et sur l'ajustement du motif de la traite aux attentes des médias ${ }^{15}$. Qu'il s'agisse de la presse écrite ou audiovisuelle, le récit mélodramatique de l'innocente jeune femme abusée par un odieux criminel et condamnée à l'asservissement sexuel dans un pays étranger n'a pas connu de transformation majeure depuis Stead. Exemple parmi d'autres, le dossier que Le Nouvel Observateur consacre le 12 novembre 2009 à «l'explosion des trafics sexuels » relatant le parcours terrifiant de la jeune moldave Irina - «silhouette élancée, traits fins, grands yeux noisettes »-qui a «sauté dans l'avion » en croyant aller « travailler sur les marchés d'Antalya, la "Riviera turque" » et qui s'est retrouvée «séquestrée dans un bordel turc, enchaînant les passes comme un fantôme ». Lui fait suite celui de sa compatriote Lena, " grande blonde à la coupe carrée » à qui un jeune homme « a fait le coup du prince charmant, lui expliquant qu'elle valait bien mieux que Chisinau, sa vie monotone et sa paie de misère. Lena y a cru très fort. Elle a atterri sur les trottoirs moscovites ». Le mystère - dont s'étonnait déjà Appleton dans sa thèse de 1903 citée plus haut - de l' "extrême naïveté de certaines filles » s'expliquerait désormais par des facteurs psychologiques, l'article avançant que « $85 \%$ des victimes de trafic ont subi des abus au sein de leur famille ${ }^{16} »$.

La récurrence de tels récits dans les publications abolitionnistes indique que le mouvement a bien saisi les vertus du storytelling comme outil de communication et de sensibilisation à sa cause (Poletta, 2006 ; Traïni, 2009) ${ }^{17}$. Chaque numéro du journal du Mouvement du Nid contient ainsi un récit, écrit à la première personne, de la descente aux enfers d'une jeune femme en situation de vulnérabilité qui a cru ou voulu croire à un avenir meilleur en s'engageant dans la prostitution ${ }^{18}$. Lorsque le récit est celui d'une étrangère, tromperie et crédulité sont convoquées pour expliquer l'acceptation d'une offre de migration qui se mue rapidement en descente aux enfers. Ainsi Raïssa, Albanaise qui rencontre un « homme normal » «enfin, je le pensais ») la convaincant «qu'à l'Ouest, [elle] pourrai[t] avoir une vie meilleure et un bon travail », n'a « pas compris » lorsque la compatriote qui l'a accueillie à Paris lui a demandé de l'accompagner Porte d'Auteuil ${ }^{19}$.

\footnotetext{
${ }^{15}$ Les organisations qui ont porté cette remobilisation et leurs discours publics sont exposés et étudiés dans Mathieu (2014).

${ }^{16}$ L'altérisation psychologique se redouble d'une altérisation culturelle dans le cas des prostituées nigérianes dont la migration serait précédée d'une cérémonie de vaudou (le «juju») destinée à les asservir par la crainte d'une sanction magique si elles cherchaient à s'émanciper de leurs exploiteurs.

17 Storytelling d'autant plus efficace, aujourd'hui comme hier, qu'il adresse une offre d'engagement (un soutien à l'abolitionnisme et à ses revendications) relevant de ce que Luc Boltanski (1993) a conceptualisé comme une «politique de la pitié ».

${ }^{18}$ Les récits sont ceux de personnes prises en charge par le Mouvement du Nid mais mis en forme par les journalistes de l'association. Précisons également que l'abolitionnisme contemporain entretient un rapport sélectif à son héritage : si Butler reste une figure révérée, celle de Stead est expurgée de l'histoire que le mouvement livre de lui-même.

${ }^{19}$ Claudine Legardinier, «Raïssa : Les clients ? Je ne veux plus jamais en parler. Plus jamais y penser », dans Prostitutionetsociété.fr. En ligne, consulté le 28 avril 2017.

URL : http://www.prostitutionetsociete.fr/temoignages/raissa-les-clients-je-ne-veux-plus.
} 
Un intense lobbying a permis au mouvement abolitionniste français d'inscrire la prostitution à l'agenda politique. Rencontrant la préoccupation du personnel politique devant la question migratoire, la mobilisation du motif de la traite s'est révélée efficace pour convaincre que des mesures avant tout d'ordre pénal étaient prioritaires pour abolir la prostitution. Non seulement les parlementaires se sont appropriés les arguments abolitionnistes lors de l'élaboration de la récente loi « renforçant la lutte contre le système prostitutionnel », mais ils en ont reproduit les formes, et spécialement un storytelling basé sur le motif de la naïve jeune femme abusée par des fausses promesses et transportée en France par un «réseau » de proxénètes. Ainsi, le chapitre consacré à la traite du rapport d'information sur la prostitution en France des députés Danielle Bousquet et Guy Geoffroy contient-il des récits à la structure narrative désormais familière, tel celui-ci :

Arrivée à l'âge de 17 ans en France, par le biais d'un réseau de traite dont elle ignorait le véritable but, Baina, sous la menace d'un couteau, a été violée puis contrainte de se prostituer. Elle rapporte qu'il lui était alors impossible d'arrêter la prostitution et de s'enfuir, tant ses proxénètes l'avaient endoctrinée. Il lui était même interdit de parler aux associations présentes sur le terrain, car cela risquait de jeter «le mauvais œil » sur la communauté toute entière ${ }^{20}$.

La conviction que des criminels organisés abusent de la faiblesse de jeunes femmes naïves pour les déplacer de leur pays d'origine et les contraindre à la prostitution constitue, on le voit, un appui pour des politiques publiques mobilisant les moyens de plusieurs administrations (police, justice, affaires sociales et droits des femmes). Pourtant, nombreux sont les travaux sociologiques, par exemple John Davies (2009), à constater des décalages entre cette structure narrative et les parcours migratoires des étrangères se prostituant en France. D'autres, relevant la rareté des condamnations pénales pour traite des êtres humains, insistent sur les logiques institutionnelles qui conduisent de l'omniprésence discursive de la victime de la traite à sa métamorphose en coupable de franchissement irrégulier de frontières (Jaksic, 2016). Qu'un tel constat paraisse in fine donner raison à la thèse de Chaumont (2009) - l'argument de la traite légitimerait une politique coercitive à l'égard des migrantes - ne parvient toujours pas à rendre compte de son efficacité. Celle-ci semble moins résider dans la capacité de mass-médias, militants abolitionnistes ou politiciens démagogues à manipuler les esprits crédules ${ }^{21}$ que dans la consolidation croisée de la légende urbaine et de la politique publique. Les deux, de fait, se conjuguent pour produire ce qu'on peut appeler, à la suite de Peter Berger et Thomas Luckmann, la «structure de plausibilité » (Berger, Luckmann, 1994: 211) de la traite, la politique publique s'appuyant sur la croyance en la vraisemblance des rapts ou duperies de jeunes femmes à fins d'asservissement sexuel tandis que celle-ci trouve une ratification dans l'autorité d'une action menée au niveau gouvernemental. De sorte que l'appréhension sociologique de la traite peut espérer échapper à l'alternative du vrai ou du faux en se déplaçant vers les «modes d'existence ${ }^{22}$ » d'un phénomène qui, pour trouble et incertain qu'il soit, n'en emporte pas moins des effets sociaux tout à fait concrets et tangibles.

\footnotetext{
${ }^{20}$ Danielle Bousquet, Guy Geoffroy, Prostitution : l'exigence de responsabilité. En finir avec le mythe du «plus vieux métier du monde », Assemblée nationale, n³334, 2011, p. $43-44$ (le récit est emprunté à Prostitution et société).

${ }^{21}$ Rien ne permet en effet de postuler que ceux qui invoquent le motif de la traite à l'appui de leurs positions abolitionnistes le feraient par pure démagogie et ne partageraient pas la croyance diffuse en la réalité des cas de jeunes filles abusées ou enlevées pour être prostituées à l'étranger - ou, dit autrement, que la crédulité serait un trait caractéristique des seules fractions défavorisées de la population.

${ }^{22}$ L'expression, récemment popularisée par Bruno Latour (2012), trouve son origine dans le travail de Luc Boltanski sur les modes d'existence du groupe des cadres (1982).
} 


\section{Bibliographie}

APPLETON P. (1903), La traite des blanches, thèse de doctorat, Paris, Arthur Rousseau éditeur.

BeRger P., LuCKMANN T. (1994), La construction sociale de la réalité, Paris, MéridiensKlincksieck.

BoltANSKI L. (1982), Les cadres. La formation d'un groupe social, Paris, Éditions de Minuit.

Boltanski L. (1993), La souffrance à distance, Paris, Métailié.

Chaumont J.-M. (2009), Le mythe de traite des blanches. Enquête sur la fabrication d'un fléau, Paris, La Découverte.

Chaumont J.-M. (2012), « Le militant, l'idéologue et le chercheur », Le Débat, n 172, p. 120130.

Chaumont J.-M., Machiels C. (dir.) (2009), Du sordide au mythe. L'affaire de la traite des blanches (Bruxelles, 1880), Louvain-la-Neuve, Presses universitaires de Louvain.

CORBIN A. (1982 [1978]), Les filles de noce, Paris, Flammarion.

DAVIES J. (2009), "My Name Is Not Natasha". How Albanian Women in France Use Trafficking to Overcome Social Exclusion (1998-2001), Amsterdam, Amsterdam University Press.

Doezema J. (2010), Sex Slaves and Discourse Masters. The Construction of Trafficking, Londres, Zed Book.

FARge A., ReVEl J. (1988), Logiques de la foule. L'affaire des enlèvements d'enfants, Paris, Seuil.

FINE G. A. (1992), Manufacturing Tales, Knoxville, University of Tennessee Press.

JAKSIC M. (2016), La traite des êtres humains en France, Paris, Éditions du CNRS.

KRINSKI C. (2013), «Introduction: The Moral Panic Concept», in C. KRINSKI (dir.) The Ashgate Research Companion to Moral Panics, Farnham, Ashgate, p. 1-14.

LATOuR B. (2012), Enquête sur les modes d'existence, Paris, La Découverte.

MAINSANT G. (2012), L’État et les illégalismes sexuels. Ethnographie et sociohistoire du contrôle policier de la prostitution à Paris, thèse de doctorat, Paris, École des hautes études en sciences sociales.

Mathieu L. (2014), La fin du tapin. Sociologie de la croisade pour l'abolition de la prostitution, Paris, F. Bourin.

MORIN E. (1969), La rumeur d'Orléans, Paris, Seuil.

PHILIPPON O. (1958), Un grave danger pour la jeunesse du monde : la traite des êtres humains, Paris, Tréqui. 
Polletta F. (2006), It Was Like a Fever. Storytelling in Protest and Politics, Chicago, University of Chicago Press.

STEAD W. T. (1885), Les scandales de Londres, Paris, Dentu.

TRAïNI C. (dir.) (2009), Émotions... mobilisation!, Paris, Presses de Sciences Po.

Walkowitz J. (1992), City of Dreadful Delight, Londres, Virago. 